\title{
Surface Morphology of GaN Surfaces during Molecular Beam Epitaxy
}

\author{
R. M. Feenstra, Huajie Chen, V. Ramachandran, and C. D. Lee \\ Department of Physics, Carnegie Mellon University, Pittsburgh, Pennsylvania 15213 USA \\ A. R. Smith \\ Department of Physics and Astronomy, Ohio University, Athens, Ohio 45701 USA \\ J. E. Northrup \\ Xerox Palo Alto Research Center, 3333 Coyote Hill Road, Palo Alto, California 94304 USA \\ T. Zywietz and J. Neugebauer \\ Fritz-Haber-Institut der Max-Planck-Gesellschaft, Faradayweg 4-6, D-14195 Berlin, Germany \\ D. W. Greve \\ Department of Electrical and Computer Engineering, Carnegie Mellon University, Pittsburgh, \\ Pennsylvania 15213 USA
}

\begin{abstract}
The reconstruction and surface morphology of gallium nitride (0001) and $(000 \overline{1})$ surfaces are studied using scanning probe microscopy and reflection high-energy electron diffraction. Results for bare GaN surfaces are summarized, and changes in the surface structure and morphology due to co-deposition of indium or magnesium during growth are discussed.
\end{abstract}

\section{Introduction}

Work over the past several years has identified the two families of surface reconstructions associated with the two c-plane surfaces of hexagonal GaN: the (0001) surface and the $(000 \overline{1})$ surface [1-3]. Reconstructions on both types of surfaces are quite remarkable in that they consist of metallic layers of gallium, bonded onto the GaN. This is an unusual situation for two reasons: first, most semiconductor surfaces prefer to be non-metallic, since the opening up of a surface gap provides a mechanism for energy lowering of the system. Second, the separation of the Ga atoms in bulk Ga is typically $2.7-2.8 \AA$, considerably smaller than the lattice constant of GaN, $3.19 \AA$. Nevertheless, at least for the $(000 \overline{1})$ surface, the Ga atoms on the surface form a $1 \times 1$ structure with a Ga-Ga separation of $3.19 \AA$, as pictured in Fig. 1(a). Additional reconstructions, with $3 \times 3,6 \times 6$, and c $(6 \times 12)$ symmetry, are produced by depositing sub-monolayer quantities of Ga onto this $1 \times 1$ surface, as pictured in Fig. 1(b). The additional Ga adatoms are bound on top of the $1 \times 1$ Ga adlayer, stabilized by an $\sim 0.5 \AA$ lateral relaxation of the underlying Ga atoms as seen in Fig. 1(b). The binding of these additional $\mathrm{Ga}$ adatoms is nevertheless relatively weak, and consequently these higher order reconstructions are stable only below $\sim 300^{\circ} \mathrm{C}$ above which they undergo reversible order-disorder phase transitions [4].

For the (0001) surface, in the N-rich limit, we find that the surface is terminated with an arrangement of adatoms with each adatom forming three bonds to underlying $\mathrm{Ga}$ atoms, as pictured in Fig. 1(c). Probably both $\mathrm{N}$ and Ga species are involved as adatoms; several different structures have been observed including $2 \times 2,5 \times 5$, and $6 \times 4$, with the former two believed to consist of simple adatom arrangements and the latter one apparently being a more complicated reconstruction [5]. (For the $2 \times 2$, considerable confusion exists in the literature since some reports of this reconstruction involve, we believe, arsenic contamination of the surface $[5,6])$. In the Ga-rich limit, we find 
that there are two monolayers of Ga on top of the Ga-terminated bilayer [2]. Because the bonding between two Ga layers is only weakly dependent on the registration between the layers, the Ga atoms in the top adlayer are able to adopt a more optimal lateral spacing $(\sim 2.7 \AA)$, and thereby realize the energy benefit of stronger Ga-Ga bonding within the adlayer [2,7]. An incommensurate reconstruction thus forms, as pictured in Fig. 1(d); we refer to this structure as " $1 \times 1$ " (in quotation marks) or pseudo- $1 \times 1$. This situation is in contrast to the $(000 \overline{1})$ surface, where the highly direction-dependent strength [1] of the N-Ga bond leads to a strong preference for the top site registration [shown in Fig. 1(a)] over alternative registrations of the adlayer. Therefore, incommensurate structures are not expected to occur on the $(000 \overline{1})$ surface.

In this paper we discuss the effects of the surface reconstructions on the surface morphology of growing films, for bare and adsorbate-covered GaN surfaces. Results for the bare surface are found to differ compared to those obtained with indium or magnesium overlayers; data for each case is presented, and the effects of the overlayers on the growth kinetics is discussed.

\section{Experimental}

Details of the growth are similar to those previously given [1-3], and will be briefly summarized here. GaN films are grown by molecular beam epitaxy (MBE) at a temperature of typically $700^{\circ} \mathrm{C}$ using a gallium effusion cell and rf-plasma nitrogen source. $\mathrm{N}$-polar films are grown on sapphire, and $\mathrm{Ga}$-polar films are grown on $\mathrm{Si}-$ polar $6 \mathrm{H}-\mathrm{SiC}(0001)$ substrates. The $\mathrm{SiC}$ substrates are prepared ex-situ by hydrogen etching [8]. InGaN films are grown at temperatures around $650^{\circ} \mathrm{C}$, using an effusion cell for the indium source. Typical growth rates for $\mathrm{GaN}$ and $\mathrm{InGaN}$ are $200 \mathrm{~nm} / \mathrm{h}$. Magnesium is supplied by an effusion cell with typical cell temperature of $375^{\circ} \mathrm{C}$. $\mathrm{Ga}$, In and $\mathrm{Mg}$ flux rates are calibrated with an in situ crystal thickness monitor, and the substrate temperature is measured by an optical pyrometer.

\section{Growth kinetics on bare surfaces}

Reflection high energy electron diffraction (RHEED) observation during MBE growth of GaN reveals a characteristic behavior as one varies between growth under relatively Ga-rich and N-rich conditions. In the former, the RHEED pattern is streaky, indicative of flat morphology, and in the latter the RHEED pattern is spotty, indicative of rough surface morphology. This behavior has been noted by a number of authors $[9,10]$, and indeed, the transition from smooth to rough is generally used as a definition of Ga-rich compared to N-rich growth. Atomic force microscopy (AFM) imaging has been used to image this smooth to rough behavior in real-space [10]; our results for this morphological change are pictured in Fig. 2. Figure 2(a), shows the result for $\mathrm{a} \approx 0.3 \mu \mathrm{m}$ thick film grown under Ga-rich conditions. The large-scale morphology seen there, with pits and trenches, arises from dislocations in the film, whereas on the small scale the morphology is flat as evidenced by the streaky RHEED pattern shown in the inset. Figure 2(b) gives results for the same sample which was overgrown by $0.1 \mu \mathrm{m} \mathrm{GaN}$ deposited under Ga-rich conditions (during which time the RHEED pattern was streaky, as in the inset of Fig. 2(a)), followed by $\approx 15 \mathrm{~nm}$ GaN deposited under N-rich conditions. The overall surface morphology (dominated by the effects of dislocations) is similar to that of Fig. 2(a), but on a $30 \mathrm{~nm}$ length-scale a distinct roughness of the surface can be seen and this roughness give rise to the spotty RHEED pattern shown in the inset. The results shown in Fig. 2 are for Ga-polar films, although very similar results have been obtained for the case of N-polar films [11].

An explanation for the smooth to rough behavior has been advanced by Zywietz et al. [12]. 
They consider diffusion barriers for $\mathrm{Ga}$ and $\mathrm{N}$ atoms on both (0001) and (000 $\overline{1})$ surfaces, using first-principles theoretical computations. They find firstly that the diffusion barriers are substantially less for $\mathrm{Ga}$ atoms compared to $\mathrm{N}$ atoms. Furthermore, the barrier for the faster moving species $(\mathrm{Ga})$ is substantially increased on a N-terminated surface compared to a Ga-terminated surface. The latter result would lead to the roughening behavior seen in experiment, assuming that the surface is terminated by at least some $\mathrm{N}$ under $\mathrm{N}$-rich conditions. However, this assumption is, at least for the $(000 \overline{1})$ surface, not consistent with either experiment or theory since they both find a N-terminated bilayer to be energetically unstable [1]. Nevertheless, it is suggested by Zywietz et al. that a kinetic accumulation of $\mathrm{N}$ during growth may occur on the surface, since the diffusion rate of $\mathrm{N}$ is sufficiently slow that it may not have time to find another $\mathrm{N}$ atom thereby forming $\mathrm{N}_{2}$ and desorbing from the surface. In this case, the surface concentration of $\mathrm{N}$ would indeed build up during growth, thereby leading to the reduction in the Ga diffusion rate and the corresponding onset of rough growth.

\section{Indium-covered surfaces}

As discussed above, during MBE growth of GaN, the surface undergoes a transition from smooth to rough morphology when the growth condition is switched from Ga-rich to N-rich. It has been reported that indium atoms serve as a surfactant, keeping the growth in the smooth regime when the gallium flux is slightly reduced below the transition flux [13]. However, in our earlier growth of InGaN on $\mathrm{GaN}(000 \overline{1})$, such a surfactant effect was not observed [14]. Instead we found that indium atoms had little effect on the smooth/rough transition, possibly due to the different polarity of $\mathrm{GaN}$ used in that case. Below, we report on the smooth/rough transition of InGaN growth on both the $(000 \overline{1})$ and $(0001)$ faces.

We find a dramatic difference in the smooth/rough behavior between the $(000 \overline{1})$ and (0001) faces, as shown in Figs. 3(a) and (b) respectively. For both experiments, the nitrogen flux was kept constant. Then, a certain indium flux was applied, and gallium flux was adjusted to find the smooth/rough transition point. For comparison, dashed lines in Fig. 3 show where the total metal flux (indium + gallium) is constant. Figure 3(a) shows that for the $(000 \overline{1})$ face, even when a large indium flux is applied, the gallium flux can only be reduced slightly before the growth becomes rough. In contrast, for the (0001) face, it is found that when the indium flux is applied the gallium flux can be greatly reduced (by an amount considerably greater than that of the added indium flux) before the growth becomes rough. Thus, indium serves as a surfactant for the (0001) surface.

We believe the different behavior of the (0001) and (000 $\overline{1})$ surfaces seen in Fig. 3 can be correlated with the different metal (In and Ga) content of the two surfaces - 1 ML for the (000 $\overline{1}$ ) surface and about 2 ML for the (0001) surface [14-16]. The excess metal present in the latter case may act to prevent any accumulation of nitrogen, thus preventing the associated reduction in $\mathrm{Ga}$ diffusivity. In addition, however, there is a separate mechanism which will act to increase the $\mathrm{N}$ diffusivity when $2 \mathrm{ML}$ of metal is present: we find that the equilibrium position of the $\mathrm{N}$ atoms in this case is between the metal layers and the resulting diffusivity for the $\mathrm{N}$ is significantly enhanced compared to that when the $\mathrm{N}$ resides above the top layer [17]. Thus, we expect for both $\mathrm{GaN}$ and InGaN, under metal rich conditions, faster diffusion due to both the enhanced $\mathrm{N}$ diffusivity and the prevention of $\mathrm{N}$ accumulation. These mechanisms provide an interpretation of the kinetics which is in agreement with experiment for both the clean and In-covered surfaces, of both polarity.

\section{Magnesium-covered surfaces}


We have studied the effect of $\mathrm{Mg}$ exposure on surface morphology of GaN(0001) surfaces [18]. A surfactant effect of $\mathrm{Mg}$ is seen in the N-rich regime. Exposing the growth surface to as little as 0.2 ML of Mg under Ga-poor conditions leads to a reversal of the RHEED pattern from spotty to streaky. Also, when the growth is made very N-rich by reducing the Ga flux to about one half of that at the transition point, exposure to $\mathrm{Mg}$ often produces a streaky $2 \times 2$ pattern. Figure 4 shows AFM images which present a sequence of surface morphologies during the smoothing of the surface. Figure 4(a) shows a rough film similar to the one in Fig. 2(b) grown in the N-rich regime. An exposure of about 0.2 ML of Mg leads to a film which shows areas such as those in Fig. 4(b). As indicated by the arrows, regions of 2-D growth start to nucleate on the rough film, where we can see atomic steps again. At about 0.7 ML Mg exposure, the surface is mostly smoothed out and the film shows morphology as in Fig. 4(c) and (d), which are images acquired from widely separated locations on the same sample. The morphology of Fig. 4(d) is indistinguishable from that of Fig. 2(a), but small areas of roughness are still seen in Fig. 4(c). Closer inspection reveals that these patches of roughness follow the directions of steps, suggesting that the smoothing is a step-flow process.

We believe that the presence of the Mg allows the surface to satisfy electron counting, leading to completely filled or completely empty surface state bands, for structures containing either 1/4 ML [19] or 3/4 ML [18] of Mg substituting for Ga. Qualitatively, one expects such a surface to be non-reactive, and have relatively low diffusion barriers compared to the bare GaN surface. Hence, a surfactant effect (smooth, 2-D growth) could be expected, although more detailed computations are required to confirm this expectation.

\section{Conclusions}

In summary, we find first of all that the surface kinetics of bare GaN surfaces are dominated by the presence or absence of surface Ga layers. Most reconstructions of the surface do contain terminating Ga layers, leading to fast surface diffusion and smooth morphology during growth under Garich conditions. For growth under N-rich conditions, it is believed that $\mathrm{N}$ can accumulate on the surface, giving rise to the observed rough morphology in that case. Indium adsorbates are found to effectively substitute for the surface gallium during growth of InGaN, because of the strong surface segregation of the indium. The number of metal (In or Ga) layers on the InGaN surface is found to correlate with the occurrence of smooth morphology, indicating the effectiveness of multiple metal layers in enhancing the $\mathrm{N}$ diffusivity. Finally, for $\mathrm{Mg}$ adsorbates, changes in growth kinetics are tentatively attributed to a reduction in diffusion barriers since those surface reconstructions satisfy electron counting.

\section{Acknowledgments}

This work was supported by grants from the Office of Naval Research, N00014-96-1-0214, and the National Science Foundation, DMR-9615647.

[1] A. R. Smith, R. M. Feenstra, D. W. Greve, J. Neugebauer, J. E. Northrup, Phys. Rev. Lett. 79, 3934 (1997).

[2] A. R. Smith, R. M. Feenstra, D. W. Greve, M. S. Shin, M. Skowronski, J. Neugebauer, and J. Northrup, J. Vac. Sci. Technol. B 16, 2242 (1998).

[3] A. R. Smith, R. M. Feenstra, D. W. Greve, M. S. Shin, M. Skowronski, J. Neugebauer and J. Northrup, Surf. Sci. 423, 70 (1999). 
[4] A. R. Smith, R. M. Feenstra, D. W. Greve, J. Neugebauer, and J. Northrup, Appl. Phys. A 66, S947 (1998).

[5] V. Ramachandran, C. D. Lee, R. M. Feenstra, A. R. Smith, and D. W. Greve, Phys. Rev. Lett. 84, 4014 (2000). [Comment on Q. K. Xue, Q. Z. Xue, R. Z. Bakhtizin, Y. Hasegawa, I. S. T. Tsong, T. Sakurai, and T. Ohno, Phys. Rev. Lett. 82, 3074 (1999)].

[6] V. Ramachandran, C. D. Lee, R. M. Feenstra, A. R. Smith, J. E. Northrup, and D. W. Greve, J. Cryst. Growth. 209, 355 (2000).

[7] J. E. Northrup, J. Neugebauer, R. M. Feenstra, and A. R. Smith, Phys. Rev. B 61, 9932 (2000).

[8] V. Ramachandran, M. F. Brady, A. R. Smith, R. M. Feenstra and D. W. Greve, J. Electron. Mater. 27, 308 (1998).

[9] R. Held, D. E. Crawford, A. M. Johnson, A. M. Dabiran, and P. I. Cohen, J. Electron. Mater. 26, 272 (1997); R. A. Held, G. Nowak, B. E. Ishaug, S. M. Seutter, A. Parkhomovsky, A. M. Dabiran, P. I. Cohen, I. Grzegory, and S. Porowski, J. Appl. Phys. 85, 7697 (1999).

[10] E. J. Tarsa, B. Heying, X. H. Wu, P. Fini, S. P. DenBaars, and J. S. Speck, J. Appl. Phys. 82, 5472 (1997).

[11] A. R. Smith, V. Ramachandran, R. M. Feenstra, D. W. Greve, A. Ptak, T. H. Myers, W. L. Sarney, L. Salamanca-Riba, M. -S. Shin and M. Skowronski, MRS Internet J. Nitride Semicond. Res. 3, 12(1998).

[12] T. Zywietz, J. Neugebauer, and M. Scheffler, Appl. Phys. Lett. 73, 487 (1998).

[13] F. Widmann, B. Daudin, G. Feuillet, J.-L. Rouviére, and N. Pelekanos, Appl. Phys. Lett. 73, 2642 (1998).

[14] H. Chen, A. R. Smith, R. M. Feenstra, D. W. Greve, and J. E. Northrup, MRS Internet J. Nitride Semicond. Res. 4S1, G9.5 (1999).

[15] H. Chen, R. M. Feenstra, J. E. Northrup, T. Zywietz, J. Neugebauer, and D. W. Greve, submitted to J. Vac. Sci. Technol.

[16] On the InGaN $(000 \overline{1})$ surface, the most In-rich structure is the 1ML In adlayer structure analogous to that shown in Fig 1(a). Structures containing additional In adatoms on top of this adlayer [analogous to the $3 \times 3,6 \times 6$ and $c(6 \times 12)$ ] are not seen because the large size of the In atom renders these structures unstable with respect to In desorption or In droplet formation. Thus, if a high $\mathrm{N}$ diffusivity requires the presence of additional adatoms on top of the adlayer, the replacement of the Ga by $\mathrm{In}$ in the adlayer will lead to a reduction in the $\mathrm{N}$ diffusivity and therefore lead to rough growth.

[17] T. Zywietz, J. Neugebauer, M. Scheffler, J. E. Northrup, H. Chen, and R. M. Feenstra, to be published.

[18] V. Ramachandran, R. M. Feenstra, J. E. Northrup and D. W. Greve, to appear in MRS Internet J. Nitride Semicond. Res.

[19] C. Bungaro, K. Rapcewicz, and J. Bernholc, Phys. Rev. B 59, 9771 (1999). 
(a)

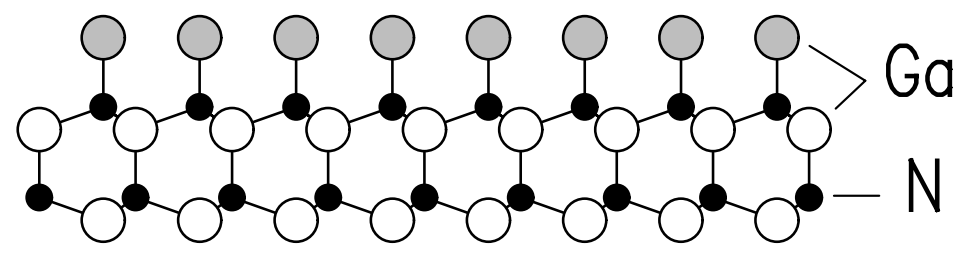

$\uparrow(000 \overline{1})$

(b)

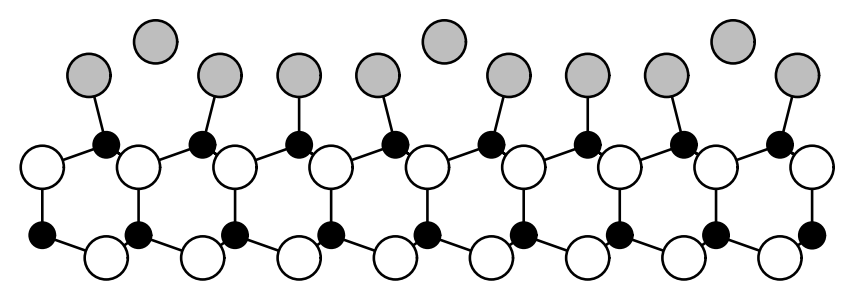

(c)

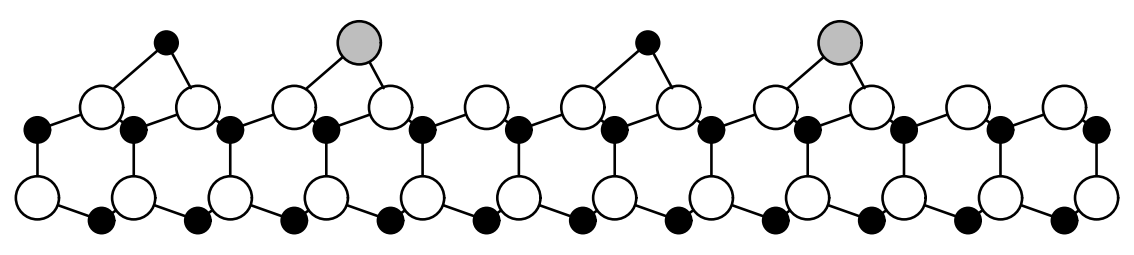

$\uparrow(0001)$

(d)

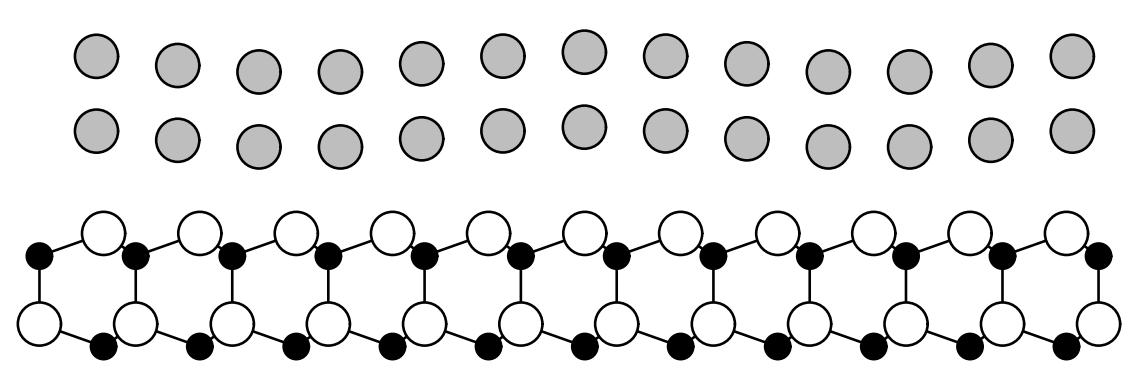

Figure 1. Schematic illustration of surface reconstructions occurring on GaN (0001) and (000 $\overline{1})$ surfaces, in the N-rich and the Ga-rich limits. (a) Ga adlayer forming $1 \times 1$ structure on the $(000 \overline{1})$ surface; (b) additional Ga adatoms forming weakly bound arrangements on this $1 \times 1 \mathrm{Ga}$ adlayer; (c) an arrangement of $\mathrm{Ga}$-adatoms and $\mathrm{N}$-adatoms on the (0001) surface; (d) incommensurate double layer of $\mathrm{Ga}$ adatoms on the (0001) surface (the vertical displacements in the double layer are shown exaggerated, for clarity). 


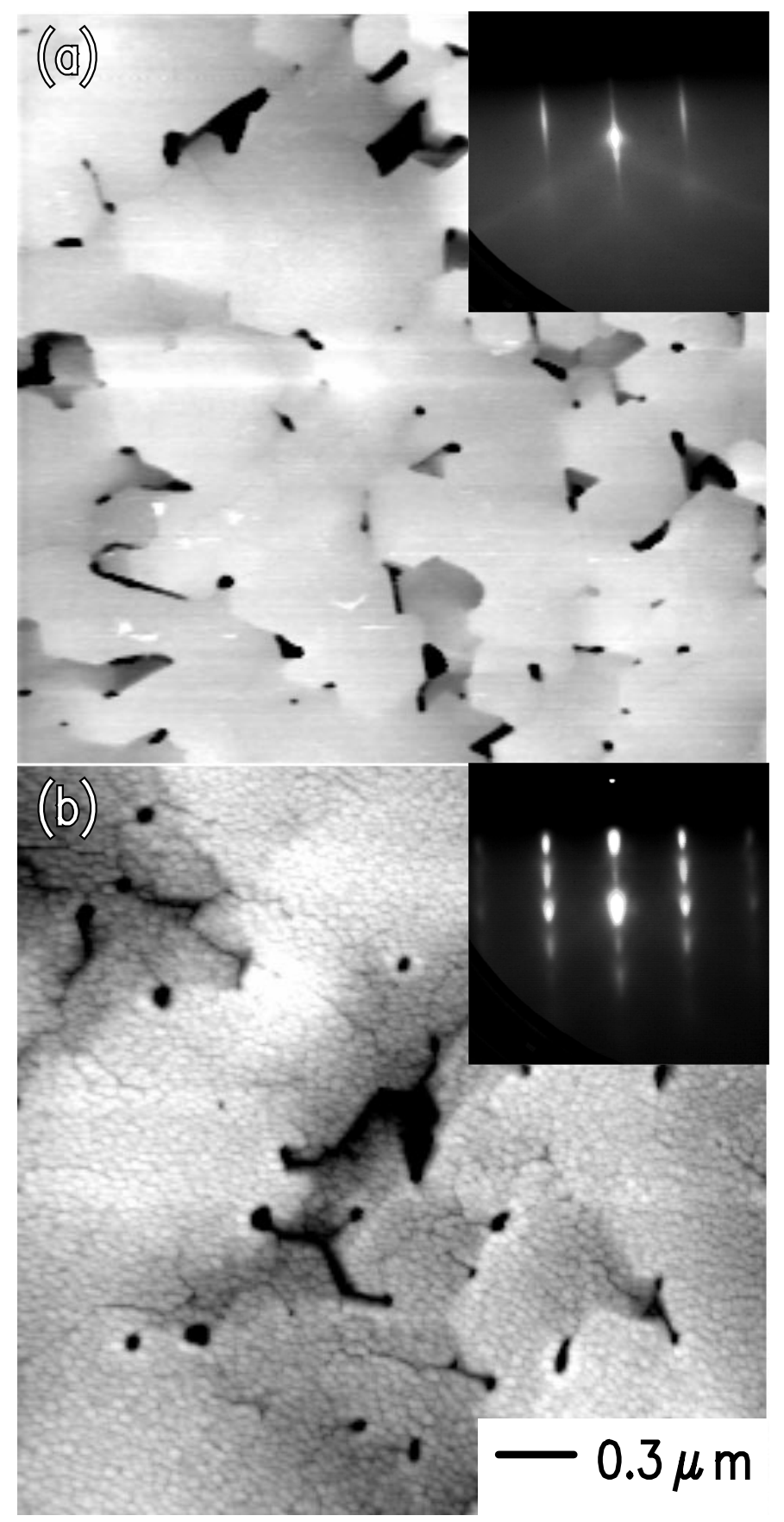

Figure 2. AFM images of $\mathrm{GaN}(0001)$ films on $\mathrm{SiC}$ : (a) grown under Ga-rich condition, and (b) grown under N-rich conditions. Grey-scale range for both images is $13 \mathrm{~nm}$. The insets show the RHEED patterns for each film, taken with the electron beam along a [11 20$]$ direction. 


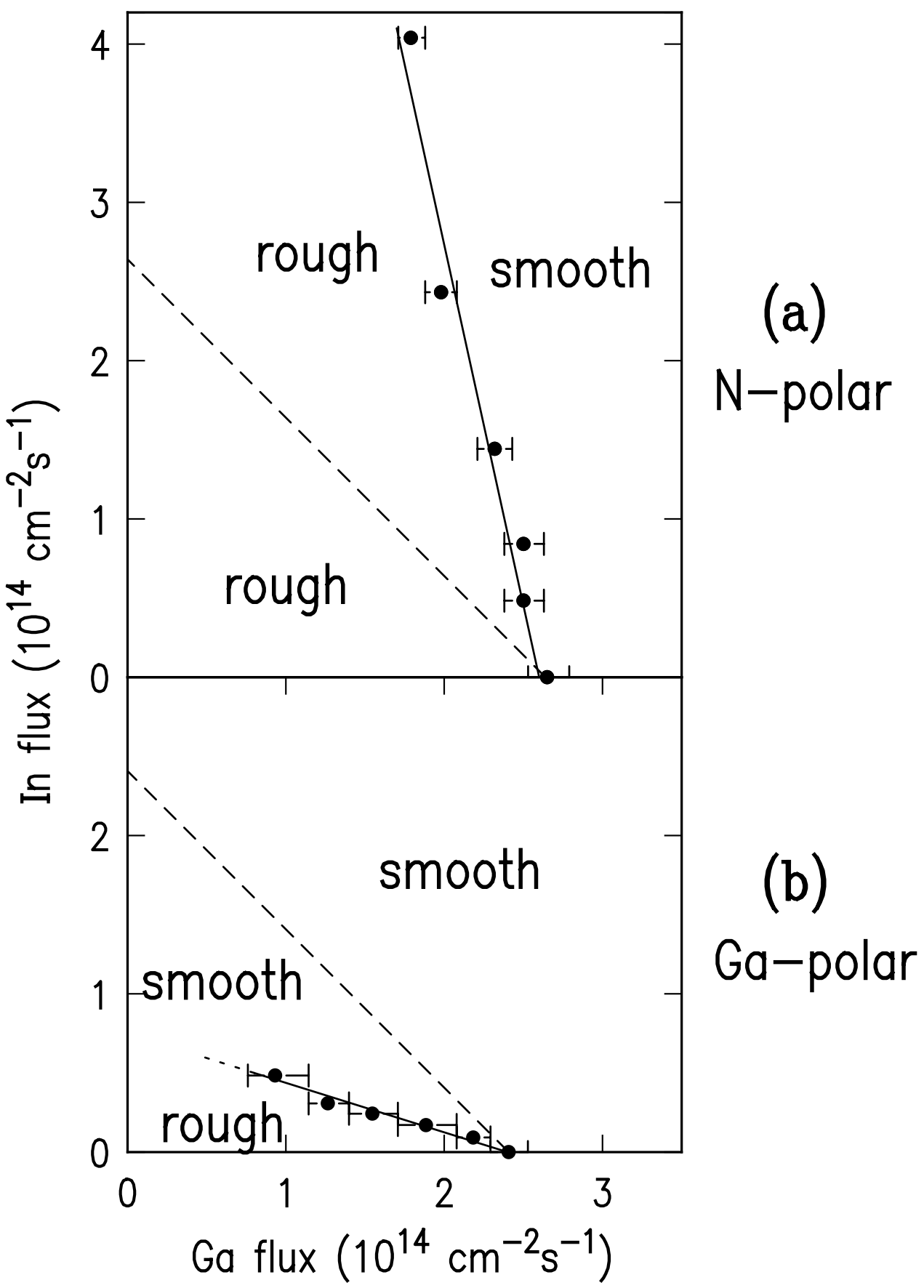

Figure 3. Smooth/rough transition on (a) (000 $\overline{1})$ face (N-polar) and (b) (0001) face (Ga-polar). Nitrogen flux was fixed in both experiments. Substrate temperature was $600^{\circ} \mathrm{C}$. Experimental data is shown with dots, each with an error bar. A dashed line is shown in each figure for comparison denoting the line with constant total metal flux. To the right of the transition lines (solid lines) the growth is smooth. 


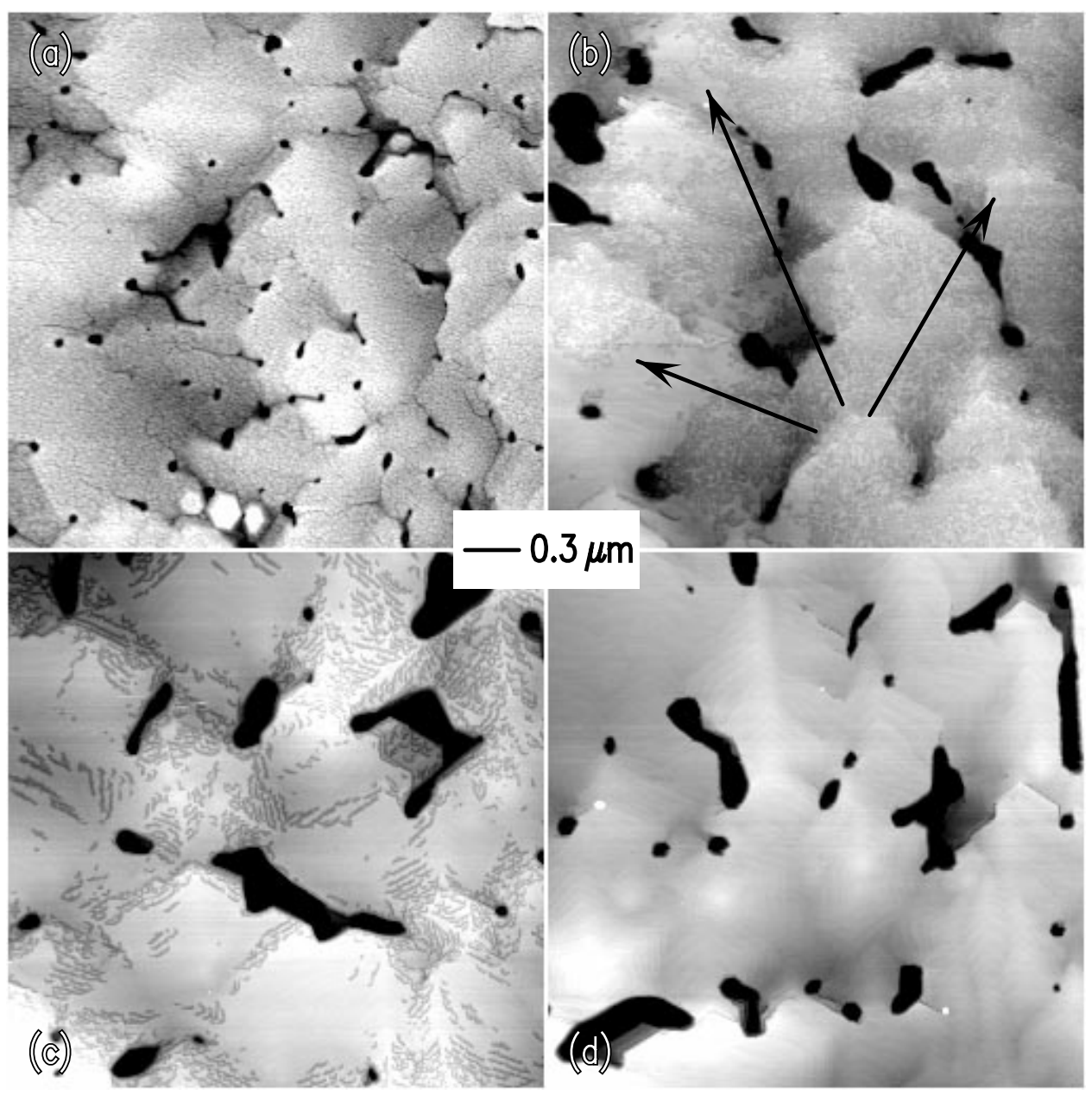

Figure 4. AFM images showing the surfactant effect of Mg: (a) GaN(0001) film grown in N-rich regime without $\mathrm{Mg}$, (b) and (c) intermediate stages of the smoothing process, (d) complete smoothing of surface as a result of $\mathrm{Mg}$ exposure. The arrows in (b) point to smooth areas beginning to appear interspersed amidst the rough regions. Grey-scale range is $16 \mathrm{~nm}$ for all images. 